Relations industrielles

Industrial Relations

\title{
Salaire et marché du travail interne à l'entreprise, par Jean-Pierre d'Aubigney, Montréal, École de relations industrielles, Université de Montréal, 1978, 51 pp.
}

\section{Jacques Mercier}

Volume 34, numéro 1, 1979

URI : https://id.erudit.org/iderudit/028954ar

DOI : https://doi.org/10.7202/028954ar

Aller au sommaire du numéro

Éditeur(s)

Département des relations industrielles de l'Université Laval

ISSN

0034-379X (imprimé)

1703-8138 (numérique)

Découvrir la revue

Citer ce compte rendu

Mercier, J. (1979). Compte rendu de [Salaire et marché du travail interne à l'entreprise, par Jean-Pierre d'Aubigney, Montréal, École de relations industrielles, Université de Montréal, 1978, 51 pp.] Relations industrielles / Industrial Relations, 34(1), 209-210. https://doi.org/10.7202/028954ar

Tous droits réservés @ Département des relations industrielles de l'Université Laval, 1979
Ce document est protégé par la loi sur le droit d'auteur. L'utilisation des services d’Érudit (y compris la reproduction) est assujettie à sa politique d'utilisation que vous pouvez consulter en ligne.

https://apropos.erudit.org/fr/usagers/politique-dutilisation/ 
provenant tantôt de la Régie Renault, tantôt des autres entreprises françaises et d'études gouvernementales relativement récentes. L'interprétation faite de tel ou tel tableau particulier doit donc être prudente, mais le lecteur en est prévenu par les auteurs, et leur usage des informations disponibles n'empêche en rien l'atteinte de leur objectif qui est de présenter "une problématique générale des inter-relations entre les différentes variables... ainsi que l'identification des principales tendances d'évolution de ces variables".

Voici quelques points saillants de l'étude. L'industrie française de construction automobile a produit en 1975, 2,54 millions de véhicules pour particuliers: un chiffre qui représentait $10,1 \%$ de la production mondiale et situait la France au quatrième rang parmi les pays constructeurs. Toujours intensive en utilisation de la main-d'œuvre, l'industrie française a eu tendance à accentuer progressivement la substitution de capital de façon à réduire, à la longue, la qualification des emplois. Dans un même élan, l'industrie a eu tendance à se décentraliser géographiquement afin de bénéficier d'une main-d'œuvre susceptible de mieux répondre aux exigences de ces emplois, c'est-à-dire une maind'œuvre non-industrielle, mais de préférence nationale plutôt qu'étrangère.

Les conclusions et les perspectives d'avenir sont basées sur des considérations réalistes concernant les marchés du produit, la productivité du travail, et la durée du travail. Le tout laisse entendre que l'industrie survivra, mais devra revêtir des formes nouvelles dans sa phase de maturité.

Le rapport ayant donc une application toute particulière à la France présente néanmoins un cadre d'analyse précieux à tous ceux qui s'intéressent à l'industrie automobile, ainsi qu'aux marchés du travail en général. De plus, ceux qui s'intéressent aux applications pratiques de l'organisation scientifique du travail et à la discrimination au travail y trouveront des considérations implicites qui s'avèrent intéressantes.

Kevin O'FARRELL

Université Laval
Salaire et marché du travail interne à l'entreprise, par Jean-Pierre d'Aubigney, Montréal, École de relations industrielles, Université de Montréal, 1978, $51 \mathrm{pp}$.

Pour qui s'intéresse de près ou de loin aux aspects économiques des relations industrielles, la distinction entre marché externe (i.e. dont le recrutement se fait auprès de la main-d'œuvre extérieure à l'entreprise) et marché interne (i.e. où les promotions et transferts sont comblés généralement à partir des employés de l'entreprise) revêt un caractère analytique appréciable. D'ailleurs, les origines de ce type d'application à la structuration et au fonctionnement des marchés du travail se trouvent dans la tradition appelée «institutionnaliste " dont on connaît surtout les travaux de certains économistes du travail américains tels Reynolds, Ken, Dunlop, Lester, Ross. Et l'un des principaux développements de cette tradition est sans contredit la notion de système de relations industrielles à laquelle s'intègre d'ailleurs passablement bien celle de marché interne du travail.

Dans cette monographie, le professeur Jean-Pierre D'Aubigney nous présente, du point de vue de l'analyse micro-économique orthodoxe, l'essentiel de cette théorie. L'étude comporte trois parties à savoir 1) fondements et caractéristiques du marché interne (les trois premiers chapitres), 2) critique du fondement théorique de l'approche (chapitre 4 et 3 ) proposition alternative sur le fondement du marché interne (chapitre 5). L'ouvrage est bien fait, il est clair, rigoureux, synthétique et demeure facilement accessible par le non-économiste.

La décision de restreindre l'exposé au point de vue néo-classique présente l'avantage d'explorer plus à fond l'apport de la théorie économique sur le phénomène des marchés internes et d'en cerner les possibilités et les limites. C'est justement à ce type d'exercice que l'auteur se livre dans les parties 2) et 3 ). Non seulement comme le souligne à juste titre l'auteur, la spécificité technologique est un concept trop restrictif pour donner lieu à une théorie de l'internalisation mais l'explication alternative proposée, la lutte contre la mobi- 
lité volontaire du salarié et de son coût, est elle-même limitée.

Il faudra sans doute ré-introduire certains éléments de l'analyse «institutionnaliste" tels la relation entre formation en cours d'emploi et processus de socialisation dans le milieu de travail, instabilité d'emploi et organisation (formelle ou informelle) du «job temtory», salaires et structures sociales de rémunération.

\section{Jacques MERCIER}

Université Laval

Le fondateur du syndicalisme catholique au Québec, Mgr Lapointe, par Robert Parisée, Montréal, Les Presses de l'Université du Québec, 1978, 80 pp.

Mgr Eugène Lapointe est certainement une grande personnalité qui a imprimé sa marque sur le développement de la pensée et de l'action sociales de la région du Saguenay au début du siècle. Son zèle, son dévouement pour l'amélioration de la condition des classes laborieuses ont inspiré beaucoup d'autres apôtres sociaux à œuvrer dans ce domaine. Il est devenu un personnage légendaire.

L'auteur nous avertit que «son ouvrage a pour objectif d'exposer systématiquement la pensée et l'action de $\mathrm{Mgr}$ Eugène Lapointe dans le domaine syndical exclusivement" et qu'il repose, pour l'essentiel sur un travail de recherche effectué aux archives de la Société historique du Saguenay.

Six très brefs chapitres constituent l'ouvrage. Ceux qui ont connu $\mathrm{Mgr}$ Lapointe resteront sur leur appétit. Quant aux autres, je crains qu'ils ne pourront jamais s'expliquer pourquoi Mgr Lapointe a pu jouir d'une telle réputation.

Ce n'est pas une étude fouillée ni systématique de la pensée du personnage. On n'y apprend pas beaucoup plus que ce que l'on trouve dans l'article de Michel Têtu publié dans Relations industrielles en 1962.

Faute d'être replacées dans leur contexte idéologique et historique, les conceptions et les positions de Mgr Lapointe peuvent difficilement être comprises par le lecteur contemporain et risquent plutôt de discréditer le personnage et son auvre.

Répéter aujourd'hui que Mgr Lapointe a été le fondateur du syndicalisme catholique au Québec, ce n'est pas simplement une exagération mais c'est une erreur. En effet, malgré les bonnes intentions et le dévouement de son fondateur, la Fédération ouvrière de Chicoutimi de 1907 n'était pas à proprement parler du syndicalisme, mais simplement une société mutuelle dont l'efficacité a été douteuse et dont la durée a été éphémère. Constater cette vérité, ce n'est pas une injure à la mémoire de $\mathrm{Mgr}$ Lapointe; ce n'est pas non plus nier le travail qu'il a accompli, car celui-ci donnera ses fruits plus tard et la région du Saguenay deviendra un des foyers les plus actifs du syndicalisme catholique au Québec.

Il aurait été intéressant d"expliquer comment $\mathrm{Mgr}$ Lapointe en était venu à sa conception d'action ouvrière qu'il considérait comme du syndicalisme, où il avait pris ses sources d'inspiration, à quelle école de pensée il se rattachait, pourquoi il avait choisi cette école plutôt qu'une autre, quels problèmes cela lui a causé au Québec avec d'autres penseurs sociaux instigateurs du syndicalisme catholique qui appartenaient à une autre école, comme l'abbé Maxime Fortin et Alfred Charpentier, etc. Mais pour en arriver là, l'auteur aurait du pousser ses recherches au delà des archives personnelles de $\mathrm{Mgr}$ Lapointe qui, tout intéressantes qu'elles soient, m'apparaissent assez limitées.

Cet ouvrage relève beaucoup plus de la littérature édifiante que de l'histoire. Mgr Lapointe aurait mérité mieux que cela.

Gérard DION

Université Laval

The Role of Trade Unions in Developing Societies, by Everett M. Kassalow and Ikanig Damachi (Eds), Geneva, International Institute for Labour Studies, $1978,183 \mathrm{pp}$.

Les études sur le syndicalisme dans les pays en voie de développement ont 